\title{
Escuela e infancia: cambios y permanencias en una comunidad nahua tlaxcalteca
}

DOI: https://doi.org/10.32870/dse.v0i10.285

\section{Martha Areli Ramírez Sánchez*}

Resumen: Este artículo explora algunos de los cambios menos evidentes ocurridos en la concepción local sobre la infancia en una comunidad indígena campesina nahua del México Central a partir de la introducción del sistema escolar y la migración internacional. Estos cambios han generado tensiones y conflictos en la vida pública y privada de las familias tlalcualpeñas. Lo cual ha generado una reconfiguración del espacio que ocupan los niños en el grupo doméstico. Sin embargo, se encontró que el aumento del gasto familiar no ha cambiado sustancialmente las funciones de los niños, sino que solamente los ha ubicado en otro espacio desde el cual siguen contribuyendo a la reproducción del grupo doméstico. Esto demuestra que los patrones culturales nahuas son resistentes a los cambios y transformaciones de la economía local y global. Palabras clave: Escolaridad, migración, niñez, cambio y permanencia, comunidad nahua.

\begin{abstract}
This paper explores some of the less evident changes on the indigenous local ideas about childhood in a nahua community in Central Mexico. These changes are the result of introduction of school system and international migration in the last 4 decades. These phenomenon have produced internal conflicts and tension in the public and private family's life. As a result indigenous families have re-taken shape particularly regarding the place that their children must take in order to keep them participating on the socio-cultural reproduction process although the significant economic improvement. We stand that nahua cultural conception of the persona and particularly childhood proves to be resilient in the face of local and global economic changes. Keywords: Nahua Community, Schooling/Education, Migration, Childhood, Change and Continuity.
\end{abstract}

\section{Introducción}

En este artículo se exploran algunas de las transformaciones menos evidentes, pero cruciales para la reproducción social, sufridas por las familias de San Pedro Tlalcuapan, comunidad indígena campesina de origen nahua, durante el proceso de incorporación de los niños en el sistema educativo escolar. La década durante la cual se ha dado seguimiento etnográfico a este pueblo me ha permitido observar el tipo de tensiones que surgen entre la familia y la escuela en los espacios públicos y privados. Tensiones que se originan a partir del encuentro entre la concepción local de infancia y la imagen universalista del niño que paulatinamente se introducido en la comunidad en los últimos 15 años. En este documento se sostiene que son dos los principales eventos que han contribuido al encuentro de ambas concepciones de infancia. Primeramente la migración y en segundo lugar la relativamente reciente transformación del sistema educativo local.

Cabe resaltar que uno de los objetivos centrales de este trabajo es recuperar y mostrar al lector las categorías culturales locales en torno a la crianza e infancia de Tlalcuapan, en las cuales subyacen las prácticas de la vida cotidiana. Esto con el objetivo de ofrecer información etnográfica

* Doctora en Antropología Social por la Universidad de Manchester, Reino Unido. Profesor investigador. Posgrado en Antropología Social. Depto. Ciencias Sociales y Políticas, Universidad Iberoamericana, Santa Fe. Prolongación Paseo de la Reforma \#880. Delegación Álvaro Obregón, Lomas de Santa Fe. C.P. 01219, México, Distrito Federal. Tel. 52 (55) 5950-4000 ext. 4036. Fax: 52 (55) 5950-4223. Correos electrónicos: martha.ramirez@ibero.mx y areliramirez31@yahoo.com.mx 
que enriquezca el debate en torno a la infancia como un fenómeno cultural y localmente creado. De ahí que este documento se apoye fuertemente en información etnográfica, los testimonios y entrevistas recogidos a partir del año 2000 hasta la fecha. Sin embargo fue en los años 2001 y 2002 cuando se obtuvo la mayor parte de la información. Periodo en el cual se obtuvieron 31 entrevistas con la población nacida entre 1920 y 1970. De éstas se identificaron las recurrencias, los patrones más comunes en cuanto al tipo de infancia que se vivió en aquellos años. Un segundo periodo, que me ha permitido observar el cambio más profundo respecto a la apreciación de la educación en la comunidad se obtuvo en las subsecuentes visitas realizadas en los posteriores años al 2003. Estas irregulares pero frecuentes visitas me han permitido adoptar una perspectiva desde la que se identifican no sólo el cambio y la permanencia, sino también los reajustes y adaptaciones al mundo más amplio al cual la comunidad se ha interconectado.

\section{La infancia universal como construcción social}

La infancia como tema de investigación no es nueva, fue a comienzo del siglo XX cuando Freud (1901), Durkheim (1911), Piaget (1923) entre otros, crearon con sus trabajos un subcampo de estudio en desarrollo humano dedicado específicamente a la infancia. Los resultados de sus primeros estudios sentaron las bases para la creación de la imagen del infante dominante en la época moderna de las sociedades occidentales. Entre las características más evidentes de esta imagen resaltan la dependencia física, social, económica y emocional. Toda vez que éstas finalmente situaron al infante en una posición de subordinación ante el mundo adulto como demuestra Ariés (1992). En contraste, el tipo de niñez que aquí se presentará no sólo se contrapone a la imagen universalista antes referida, ampliamente difundida por organismos internacionales como UNICEF, OIT; sino que además nos permitirá ilustrar cómo el ámbito escolar es una de las arenas de conflicto y tensión entre el cambio y la continuidad de estas concepciones.

Consecuentemente este trabajo desea contribuir a la reanimada discusión sobre niñez e infancia, de la cual la antropología no ha participado de una manera contundente en comparación con la sociología, por ejemplo, al respecto se puede leer el trabajo de Alzate (2002).

Aunque Franz Boas identificó tempranamente a la infancia y adolescencia como un tema de estudio independiente, sólo hubo un trabajo sostenido por parte de la corriente de pensamiento Cultura y Personalidad, de donde surgieron los trabajos pioneros de Mead (1928) entre otros que se convirtieron en referencia obligada para el estudio de la infancia. De forma indirecta algunos otros estudios llamados "clásicos" de la antropología tocaron dicho tema, por ejemplo, Los peligros del alma de Guiteras Holmes (1965) pero hasta años más tarde.

Uno de los debates contemporáneos en torno a este tema, que deseo resaltar por vincularse directamente con el objetivo central de este documento, consiste en una auto-critica académica sobre el uso continuo de categorías colonizadoras y dominantes utilizada por diversas disciplinas para elaborar, diseñar y arrojar conclusiones universalistas sobre los niños (Schmitt, 2010). Esto 
ha tenido como resultado que los llamados países del norte determinen los aspectos y categorías a "observar" casi de manera obligada en las diversas infancias de los países del sur (Morrow, 2013). Particularmente Nieuwenhuys (2013) propone asumir una visión post-colonialista que permita evitar que los niños de sociedades no-occidentales sean evaluados, observados y definidos con categorías que fueron creadas en y para sociedades de tradiciones culturales diferentes (Najunda, 2010).

No es casual entonces que desde la década de los años 70 la Organización Internacional del Trabajo (OIT) impulse la investigación social dedicada al estudio de la infancia específicamente enfocada en el trabajo infantil. En consecuencia trabajos como los de la OIT $(1973 ; 1990 ; 1997$; 2000), Räda-Barner. 1997; 1997a; 1997b) y UNICEF (DIF; 1998 vol. 2; 1998 vol. 3) tomaron un tono alarmante denunciando el trabajo infantil en regiones particulares como América Latina, África y Oriente Medio. Interpretando las experiencias rurales y urbanas de trabajo infantil como si también estos contextos fueran culturalmente homogéneos (ver Galeana, 1991; López Limón, 1998; Estrada, 1996; Valle, 1982; Gutiérrez y Vega 1995; Cisneros, 1991 y De Teresa, 1996).

\section{El contexto socio-cultural}

San Pedro Tlalcuapan, municipio de Santa Ana Chiautempan forma parte del suroeste tlaxcalteca. Esta región cuenta con una milenaria actividad agrícola, la cual data de la época prehispánica (Moctezuma, 2013). Y a pesar de que a lo largo de los siglos las comunidades que conforman esta región han transformado sus actividades económicas centrales, "han pasado de ser carboneros pulqueros, arrieros - a obreros textiles, albañiles y sirvientes para convertirse en empleados en el sector informal o emigrantes ilegales a los Estados Unidos" (Robichaux, 2006) aún se puede reconocer en estas comunidades rasgos agrícolas los cuales se entremezclan con otras actividades económicas. Esta diversificación laboral ha conducido a transformaciones importantes en las dinámicas internas de las familias nahuas tlaxcaltecas, por ejemplo, ¿en qué se emplean los recursos familiares? ¿el número de hijos que tendrá la pareja recién formada? y por sobre todo ha conducido a transformar la idea de la persona, de lo que es y lo que debe hacer (Magazine y Ramírez Sánchez, 2007; Ramírez Sánchez, 2007).

En este contexto de cambio e integración al cual las comunidades rurales e indígenas campesinas alrededor del país nunca han sido ajenas, la familia ha reorganizado sus recursos materiales y culturales alrededor de un acelerado ritmo de migración transnacional y comercio local o regional. En el contexto cultural que nos ocupa, existe una serie de trabajos relevantes que documentan la permanencia de creencias y formas organizativas cruciales para la reproducción socio-cultural propia de la familia nahua del México central (Velásquez, 2013; Corona, 2011; Good, 2005; Regerh, 2005; Elu de Leñero, 1993, sólo por citar algunos).

Muy particularmente en el caso de Tlalcuapan la situación ha sido muy variable, por ejemplo en los primeros años del siglo XX la población atravesó por un periodo de extrema pobreza en el que la aportación de la fuerza de trabajo joven fue de suma importancia. En este tiempo la econo- 
mía familiar casi dependía del salario obtenido por el trabajo de los niños e hijos solteros. El trabajo principal consistía en ser entregados como mozos o sirvientes a familias que podían pagar por su servicio; sus destinos principales eran Santa Ana Chiautempan cabecera municipal de Tlalcuapan, Puebla, el Distrito Federal o Cuernavaca. Posteriormente en las siguientes cuatro décadas del siglo $\mathrm{XX}$, cambios sustanciales en la economía regional permitieron que las familias pudieron conservar a sus miembros más jóvenes en casa, sin embargo éstos no dejaron de trabajar. Como veremos más adelante el aumento en el ingreso de la familia campesina no cambió la situación de los niños, sólo se les ubicó en otro espacio familiar desde el cual pudieran continuar contribuyendo con el resto de la familia.

\section{La niñez en San Pedro}

El trabajo de campo sostenido en la comunidad de San Pedro me ha permitido interpretar a la infancia como una etapa en la vida del hombre en la que el niño es apreciado por sus padres, familiares y miembros de la comunidad como un ser que nace con capacidades sociales (Ramírez Sánchez, 2007). Lo cual lo hace apto para desempeñar trabajos físicos y desempeñar ciertas responsabilidades como el cuidado de hermanos menores, vender algunas mercancías, cuidar de animales de granja y de corral, entre otras actividades. En Tlalcuapan el niño no es ajeno, ni abstraído del mundo adulto. El niño tiene una vida muy activa, tanto en la organización familiar, como en las labores en el campo, las actividades domésticas, el trabajo asalariado y no asalariados. Su participación en la vida social es tal que es crucial para que el ciclo de desarrollo del grupo doméstico sea posible. Pues a pesar de que este tipo de niñez se reproduce simultáneamente en diferentes ámbitos de la vida social -tales como el simbólico, el moral, el espiritual. -es en las relaciones de reproducción social y durante sus diversas etapas en donde este proceso puede observarse con mayor precisión.

Si bien el tipo de infancia al que aquí se hace referencia es culturalmente particular, demuestra ser lo suficientemente resiliente como para ligarse a procesos y contextos sociales mucho más amplios y diversos. Con variaciones importantes. Como se verá en este documento el tipo de infancia vivido en este contexto subyace en diversas manifestaciones a lo largo de décadas y a pesar de las transformaciones que ha experimentado la comunidad.

Tlalcuapan es un pueblo con un alto índice de migración nacional e internacional. Pero a pesar de que la migración internacional se aceleró en los años 60's del siglo pasado la situación de los niños e hijos de familia, conceptos que uso como sinónimos, no ha variado en cuanto a sus funciones sociales y a su significado. Sin embargo, el cambio económico y la migración han abierto las puertas a ideas occidentales universalistas sobre la infancia, promovidas por organismos internacionales tales como UNICEF y OIT. Los programas gubernamentales estatales y federales de atención a la familia son los agentes a través de los cuales los organismos internacionales buscan promover un cambio en cuanto a la concepción de la familia, la salud, los niños. Estos nuevos patrones no han entrado a la comunidad sin generar conflicto y tensiones en la vida de las familias tlalcualpeñas. Se 
puede observar que ante ese escenario los conceptos locales y las ideas universales sobre la infancia subsisten paralelamente en dos ámbitos diferenciados. Los primeros — nociones locales - en los roles sociales de la vida familiar y comunitaria; y los segundos — ideas universales — en la vida escolar, es decir, en el kínder, la primaria y la secundaria.

\section{Antecedentes históricos y económicos de San Pedro Tlalcuapan}

Parte importante de las tierras que actualmente ocupa Tlalcuapan, originalmente formaron parte del desaparecido Rancho de San Juan, donde una parte de la población trabajó en calidad de peonaje. Otras familias libres producían pulque y carbón a pequeña escala para sobrevivir. El pulque de estos pequeños productores era vendido localmente y el carbón se vendía a las fábricas textiles de la cabecera municipal Santa Ana Chiautempan. De acuerdo con González Jácome (2008) en la zona rural del distrito de Hidalgo, ubicado en el suroeste tlaxcalteca, del cual formaba parte Tlalcuapan, las grandes propiedades privadas como ranchos y haciendas se entremezclaban con las tierras de los pueblos y sus barrios.

Según consta en el documento número 14 de la agencia municipal, Tlalcuapan fue dotado de tierra en los años de 1856 y 1878 de las fracciones de Meza y Ateconzolco. A pesar de que el historiador del pueblo comenta que los tlalcualpeños nunca fueron peones los documentos sobre la fundación del pueblo sostienen lo contrario. Así pues hasta la segunda mitad del siglo XIX la población trabajó en el rancho. González Jácome (2008) afirma que fue la aplicación de las Leyes de Reforma lo que afectó la estructura económica de los ranchos de la región. Durante el tránsito de un tipo de propiedad a otra, sus actividades económicas y de sustento variaron, así pues se dio paso a la explotación de montes y el trabajo de los niños principalmente como criaditos. Simultáneamente la liberación de las tierras y el fraccionamiento del rancho dieron paso a la pequeña propiedad, donde se cultivó con metepantles cercados por magueyes. Con esto las familias se dedicaron a la agricultura de autoconsumo de temporal. Actividad que durante la última década, comprendida entre el año 2003 y 2013, ha disminuido. A partir de entonces la familia de Tlalcuapan se ha inclinado por otras actividades económicas que permiten complementar el gasto familiar.

El impulso que recibió la actividad industrial en el Estado de Tlaxcala (García Verástegui y Pérez Salas, 1991) con la introducción del tramo ferroviario Puebla-Apizaco en 1869, coincidió con la consolidación del pueblo. Por ejemplo en Santa Ana Chiautempan se asentaron grandes e importantes industrias textiles en los años de 1876 y 1901 (Nutini y Barry, 1974). Estas mantuvieron gran actividad industrial hasta 1930 (Ramírez, 1991) año en que San Pedro fue oficialmente reconocido como pueblo, adoptando el nombre de San Pedro Tlalcuapan de Nicolás Bravo. En estos años la mayoría de las haciendas que aún existían en el estado se fraccionan de manera definitiva, liberando el resto de mano de obra acasillada (Suárez de la Torre, 1991). Dado que la consolidación del pueblo coincide con el declive de la actividad textil industrial de Chiautempan los tlalcualpeños no se emplearon en estas labores a pesar de la cercanía, ya que los separan solamente 4.5 kilómetros. 
La población no aporta más información al respecto, por lo cual podemos solamente suponer que el detrimento de la producción industrial en los años 30, así como la falta de mano de obra calificada para estas labores limitaron su participación en la actividad textil.

\section{Antes aquí no había trabajo}

Durante esos años el trabajo remunerado para la población adulta escaseó. Eran pocos los tlalcualpeños que tenían otro trabajo que no fuera el de campesinos o leñadores. Hacia 1950 la producción del pulque disminuyó gravemente quedando solamente 3 tlalchiqueros en el pueblo. Las opciones de empleo eran pocas, la agricultura de subsistencia no era suficiente para mantener a las familias, así como la exhaustiva explotación de la tierra no permitía obtener tanta producción como en los últimos años del siglo XIX, como consecuencia la tierra se erosiono. Ante esta situación las familias buscaron alternativas económicas redituables. A pesar de que uno de los objetivos más importantes de los gobiernos post revolucionarios en el estado de Tlaxcala fue fortalecer el sistema educativo estatal (Rockwell, 2007), la imperiosa necesidad económica de los tlalcualpeños orilló a que los padres optaran por enviar a sus hijos a trabajar en lugar de enviarlos a la escuela.

Gracias a las entrevistas realizadas con la población de adultos mayores se encontró por ejemplo que durante los años 20 hasta los años 50 existió una práctica común que consistía en entregar a los hijos más pequeños para el servicio doméstico de las casas más ricas de la cabecera municipal.

\footnotetext{
"Antes, aquí no había trabajo para los grandes, no había trabajo para los chicos, pero yo de niño me iba al monte a leñar y a vender la leña de casa en casa, en Santa Ana. Después mi papá me colocó de mozo en Tlaxcala, no supe cuánto ganaba porque mi papá cobraba. Me daban ropa, zapatos no muy buenos, pero servían y a veces era mejor que estar en tu casa...”. Sexo masculino, nació en 1924.
}

Esta práctica generó una figura económica importante: la servidumbre infantil, mocitos, criaditas, fueron oficios desempeñados por los hijos menores de las casas pobres. Los ingresos escasos, las parcelas de temporal, la disposición de recursos libres y el uso de todas las manos disponibles para el trabajo fueron una constante en la historia de este lugar. Por ejemplo, del total de adultos y ancianos entrevistados, hombres y mujeres, nacidos entre las décadas de los años veinte hasta los setenta, $70 \%$ tuvo al menos una experiencia de haber sido entregado en servicio doméstico y en consecuencia de no haber asistido a la primaria. Los padres cobraban los salarios, mismos que se incluían en el gasto familiar. Posteriormente a partir de la década de los 40 sucedieron dos eventos importantes que permitieron el cambio de San Pedro, primeramente, la introducción e impulso de la vida escolar y en segundo lugar la migración internacional, ambos puntos se abordarán en los apartados siguientes. 


\section{La vida escolar}

La primera escuela primaria se fundó en 1928 en el marco del proyecto de fortalecimiento del sistema educativo del gobierno del estado (Rockwell, 2007) a esta se inscribieron alrededor de 130 niñas y niños (Bello Isaías, 1994), los maestros eran y vivían en el pueblo. Si bien como se ha dicho anteriormente la construcción del sistema educativo tlaxcalteca fue una prioridad para los diversos gobiernos post-revolucionarios; este proceso osciló entre las crisis estatales y federales y entre la aplicación del programa de estudios "normativos", como señala Rockwell (2007) y las realidades locales de las comunidades tlaxcaltecas. Estas oscilaciones no fueron la excepción para la comunidad de Tlalcuapan, en el caso de los tlalcualpeños nacidos en la década de 1920 el asistir a la escuela primaria no fue una prioridad, sus innumerables necesidades económicas obligaron a la familia a destinar a los miembros más pequeños a la realización de actividades remuneradas. La escuela estaba en lo que ahora son las instalaciones del auditorio municipal en la parte central del pueblo. Al terminar la jornada escolar las primeras generaciones de estudiantes regresaban a casa a cumplir con las obligaciones que les encomendaban sus padres. La primera escuela jugó un papel importante, aunque en sus primeros años esta institución no formó parte importante de la vida y la rutina de los niños. Es decir, aún no se le encontraba una utilidad práctica en la vida de la comunidad, ya que sus prácticas educativas y contenidos se concibieron como parte de la política civilizatoria que se aplicó en todas las regiones indígenas del país (Loyo, 2006), enfrentaron cierto rechazo por parte de los padres de los primeros alumnos.

Los adultos mayores entrevistados para este capítulo fueron de los primeros tlalcualpeños que asistieron a la escuela, aunque casi ninguno dice recordar mucho de esa época. Sólo comentan que no terminaron sus estudios porque tenían que ir a ayudar a sus padres en el trabajo del campo. Otros comentaron que antes no era importante estudiar, porque el trabajo que hacían no requería de estudios, ahora dicen, es diferente. Estos informantes son generalmente bisabuelos o abuelos de los jóvenes y niños que ahora han llegado a estudiar la telesecundaria o el bachillerato tecnológico. Los hijos de estos informantes, hoy en día adultos, tampoco terminaron de estudiar la primaria. Sin embargo sus hijos sí han continuado sus estudios como ya se ha señalado. Dado el creciente número de jóvenes que asisten a las escuelas de educación superior se aprecia un cambio positivo otorgado a la educación escolarizada. Los padres de hoy dicen que es importante que los jóvenes de ambos sexos estudien una carrera técnica o universitaria, sin embargo no es la norma en el pueblo.

El padre de una familia con la que se ha mantenido contacto por más de 10 años, me comentó que sí le hubiera gustado estudiar, pero que con la gran familia que tuvo, con un salario de "profesionista", como él se refiere a las personas que han estudiado cualquier tipo de licenciatura, no le hubiera alcanzado para mantener a su familia. Que por lo tanto se hubiera tenido que casar con otra mujer que también fuera profesionista. Ahora bien, el proceso de escolarización no fue automático pero sí progresivo, por ejemplo, en el año 2000 sólo tuve conocimiento de tres familias que no permitían a sus hijos asistir a la escuela. Ante esta situación las mujeres adultas hablaban mal de 
estas familias. Poco a poco el asistir a la escuela, a la preparatoria e incluso ir a la universidad se le ha otorgado un valor positivo. Esta escolarización de la vida infantil ha traído cambios en muchos aspectos, por ejemplo, a los programas escolares le han seguido los programas de beca. En el año 2000 las familias buscaban animadamente que sus hijos fueran inscritos en el programa PROGRESA. Para su obtención, los alumnos tenían que cumplir con un registro bimestral del estado de la salud del niño. Esto significaba que debían incorporar hábitos de higiene que no eran muy comunes en el lugar, tales como: mayor frecuencia del aseo personal (ya que hasta el año 2005, la mayoría de las familias realizaban el baño solamente los sábados), mejorar la limpieza bucal.

Como se ha señalado anteriormente la introducción o imposición de ciertos hábitos de higiene formaron parte del proyecto indigenista a nivel nacional y han sido una constante en las políticas de des-indigenización de la población mexicana. Sin embargo en el caso particular de Tlalcuapan, éstos han tenido en fechas recientes además otro efecto negativo y es que ante la demanda de los maestros de llevar a la escuela papel sanitario, cepillo y pasta de dientes, un garrafón de agua, aparte del uniforme escolar y participar en los festivales escolares, la familia se ha visto en la disyuntiva de decidir quiénes de los hijos dejan de asistir a la escuela. En estos reajustes se observó que la familia ha optado por sacar a las hijas de la primaria, bajo el argumento de que "a ellas las van a mantener y no necesitan terminar la primaria.

Con la introducción de estas normas escolares las familias enfrenta situaciones nuevas y esto fue y ha sido motivo de conflictos internos. Una situación familiar me develo el tipo de conflictos que actualmente enfrenta la familia. Una tarde el tercer hijo una familia tlalcualpeña amiga mía, le dijo a su madre al volver de la escuela, que para la siguiente semana debía llevar a la escuela un regalo y vestir ropa de fiesta, porque en el salón de clases iban a celebrar el cumpleaños de un compañero de clase. La madre lavaba la ropa en el patio, comenzó reír mientras le gritó a su hija menor, "Yolanda saca el smoking de Alejandro porque lo voy a lavar para que vaya a su fiesta". Ella reaccionó de esa manera a sabiendas de que su hijo no tenía un traje de ese tipo.

Ella volteó a verme mientras decía: “¿No es tonto eso? Apenas tenemos para comer y ahora debe llevar un regalo y vestir de fiesta en la escuela. Esto es demasiado, de verdad que en la escuela se pasan". Es importante señalar que hasta hace 15 años los cumpleaños no se celebraran, o no era frecuente que esto sucediera. Pero estos cambios son alentados por los profesores, quienes también me comentaron que a lo largo de sus cursos tienen que insistir en la necesidad de que los alumnos aprendan lo que es la "disciplina". Por disciplina se refieren a aprender o incorporar hábitos de higiene personal de forma más regular, ya sea antes de asistir a la escuela o en casa. Incluso en los salones de clases los maestros exhibían ante sus compañeros de clase, a los niños que no se habían bañado o lavado los dientes. Los maestros aseguraban que con estas medidas los niños aprenderían más rápidamente lo que debían hacer.

A este respecto lo que más llamó mi atención fue que estos mismos maestros, quienes habían asumido como tarea fundamental la transmisión de estas nuevas pautas e ideologías comparten el mismo origen indígena campesino de sus alumnos. Algunos de ellos me dijeron que también eran 
campesinos y que provenían de los pueblos vecinos. Todos ellos eran maestros rurales y viajaban diariamente a San Pedro desde Santa Ana Chiautempan o incluso de más lejos. La introducción de estos patrones y la asimilación de una rutina escolar son pautas orientadas a difundir un modelo de niñez moderna. Según la cual, los niños deben recibir un trato diferenciado y más cuidados. En palabras de algunos maestros entrevistados esto significaba "el tratar a los niños como niños". Aunque desde el ámbito de la pedagogía esto ha sido señalado en trabajos como el que coordinan Baquero y Narodowski (1994), es decir el papel que juega la escuela en la construcción de la infancia, lo que permite el trabajo etnográfico es que muestra los microprocesos que permiten o no dicha construcción. Es así que podemos señalar empíricamente las diferentes tensiones al interior de las familias y entre las autoridades escolares y los padres de familia.

\footnotetext{
“Cuando yo estaba en la escuela iba con mi ropa de diario, nada de uniformes. Nos íbamos así como nos levantábamos y regresando nos íbamos al campo. Ahora es pura sacadera de dinero. Ahora quesque les piden cepillo de dientes, ropa de gala, un rollo de papel para el baño. Sí, los tiempos cambian...”. Sexo femenino, nació en 1962. Madre de 6 hijos, 5 de ellos asisten a la escuela.
}

Es difícil precisar cuando la vida escolar llegó a formar parte indispensable de la vida familiar. Lo que sí es claro es que las remesas enviadas por los migrantes favoreció la permanencia de los niños en la escuela, asimismo ha contribuido a otorgarle un valor positivo a esta práctica. La escuela y la experiencia migrante han transmitido poco a poco un discurso según el cual los "niños" son individuos dependientes, delicados, no aptos para el trabajo. Esto se refleja en los argumentos de los padres y abuelos de hoy:

"Antes nuestros padres no sabían cómo tratarnos. O ahora los niños no son como antes. Ahora cualquier cosa que les pasa y los llevan al doctor. Antes no, antes éramos más fuertes...". Sexo masculino, nació en 1936.

Según estas ideas todas las labores que realicen los niños en las que se requiere fuerza física tienen un efecto negativo en la vida de los niños. De forma progresiva los profesores han aprendido y asimilado este discurso, al menos eso dicen en su trabajo, puesto que la mayoría de ellos también son campesinos. La escuela como institución difunde lo que debe ser un niño o lo que la gente considerada moderna debe hacer con sus hijos. Según los maestros esto viene contenido en los libros de texto escolares y también se enseña en las pláticas impartidas en el centro de salud del pueblo. De tal forma que progresivamente se ha ido introduciendo en el pueblo la idea de lo que "debe ser un niño".

Por ejemplo, en el año 2000 conocí a una pareja de novios, él era campesino, pero cuando decidió que se robaría a su novia buscó otro trabajo. En el año 2001 llegó a ser obrero en una planta 
ensambladora de automóviles en Puebla. En el año 2002 esta pareja se había casado y tenido a su primer hijo varón; al preguntarles sobre lo que deseaban para el futuro de su hijo, él contestó:

"Yo quiero que mi hijo vaya a la escuela, que se prepare, que tenga otro tipo de trabajo. No quiero que sea un mugroso como yo. Quiero que sea mejor que lo que fuimos nosotros". Sexo masculino, nació en 1978.

Por otro lado, se observó que estas nuevas concepciones se enfrentan a las prácticas locales dadas las responsabilidades que conlleva la vida escolar. Por ejemplo, un maestro de la escuela primaria me comentó que los alumnos faltan a clases sobre todo en agosto y septiembre, en la temporada en que maduran las nueces ya que los niños más pequeños son ocupados en sus casas para pelarlas y venderlas en Santa Ana. En la telesecundaría sucedía algo similar, un maestro me comentó que los alumnos faltan más en la temporada de la cosecha del maíz. Para corroborar esta información pedí que me dejaran revisar las listas de asistencia de la primaria y secundaria. Primero me percaté que había un archivo incompleto y desordenado sobre el historial académico de los alumnos y las listas existentes no registraban tales faltas.

Para profundizar en este tema hablé con algunas madres que pertenecían a la sociedad de padres de familia y otras madres que no pertenecían a esta junta, pero que tienen a sus hijos inscritos en estas escuelas. Las madres comentaron que ellas iban a las escuelas a justificar las faltas de sus hijos y que los profesores justificaban estas faltas ante las autoridades. Comentaron que 3 faltas se justifican con la presentación de un trabajo escolar extra al final del curso, con la participación de sus hijos en un festival escolar o realizando faenas. Lo que significa que el alumno debe realizar trabajos de construcción, mantenimiento o limpieza de las instalaciones del plantel. Lo que en realidad contradice las ideas que los mismos maestros promueven con respecto a que los niños son delicados y deben ser tratados con cuidados especiales. Este ejemplo simple ilustra que las dos concepciones sobre lo que un niño es y debe hacer convergen a tal punto en que se tiene que negociar la coexistencia de ambas nociones. Por un lado la visión moderna escolar sobre la infancia y por otro lado la lógica domestica de las familias de San Pedro.

\section{La migración una opción para San Pedro}

En el contexto antes comentado de falta de trabajos asalariados y pobreza, en la segunda mitad del siglo XX surge para San Pedro una opción para mejorar su situación económica. En 1957 el gobierno del estado y la Secretaría de Gobernación pretendieron regular las prácticas migratorias a través de la expedición de certificados y recomendaciones para presentarse al Centro de Contratación en el estado de Sonora, México (Suárez de la Torre, 1991). Los aspirantes tenían que acreditar que no poseían bienes suficientes para el mantenimiento de su familia. El pueblo de Tlalcuapan participó de manera significativa en este proceso.

Los destinos principales de los tlalcualpeños fueron los campos de California, San José, Costa Mesa, el Condado de San Joaquín, donde se empleaban como jornaleros agrícolas con contratos 
temporales en la poda y pizca de uva, durazno, almendra, algodón, corte de espárragos, cereza y chabacano. En este tiempo de cambios y ausencias en el pueblo se requirió de mayor participación de los niños en el campo y en otros trabajos. Ellos apoyaban a sus madres y en general a sus grupos domésticos. Me comentaron algunas mujeres ahora adultas y madres de familia, que a ellas y a sus hermanos menores les tocó hebrar, hilar, deshilar, seleccionar pedazos de tela y bordar capas de lana que se vendían en Santa Ana. Ellos fueron los que realizaron estas actividades una vez que quebró la industria textilera de Santa Ana y esta industria optó por pagar destajo para que se realizaran estos trabajos.

\footnotetext{
"Cuando mi papá se fue trabajamos más, pues no estaba la cabeza de la casa, todos, en lo que pudiéramos, chicos y grandes, varias familias se quedaron así, igual que nosotros sin sus hombres, pues a trabajar más, todos...". Sexo masculino, nació en 1958.
}

Para el migrante los primeros años de trabajo en el extranjero fueron difíciles, pero la organización del trabajo familiar se mantuvo y poco a poco, fueron migrando más y más tlalcuapeños, hasta que en la actualidad se habla de que hay “otro San Pedro en Estados Unidos”. Finalmente, lo que inició como una forma de obtener dinero extra terminó por ser la principal fuente de ingresos para el pueblo. Esto marcó profundamente el destino y conformación del lugar y sus habitantes. Los grandes beneficios de la migración masiva aparecieron a finales de los años sesenta, una vez que se establecen de forma permanente los tlalcualpeños en Oxnard y Costa Mesa en California. En el año 2001, 95\% de las personas entrevistadas afirmaron tener al menos un familiar que trabajaba y radicaba allá; 94\% recibía o recibió dinero, ropa, aparatos electrodomésticos, automóviles, juguetes y otros bienes de parte de sus parientes.

\section{El Impacto de la migración}

Al revisar la historia de San Pedro podemos afirmar que las remesas o envíos de dinero han sido el principal promotor de la economía local y los principales cambios se reflejan en tres ámbitos a) en la creación de obras públicas; b) construcción y mejoramiento de las casas particulares de los migrantes; c) el establecimiento de negocios familiares y d) ha permitido la permanencia de los niños en la escuela así como que continúen con sus estudios. Todos estos cambios se han traducido en empleos para los tlalcualpeños que han decidido permanecer en el pueblo. La siguiente reconstrucción de la historia del pueblo se realizó a partir de los relatos de la población.

En 1960 se creó el primer camino oficial para conectar Tlalcuapan con Santa Ana Chiautempan. Ya existía uno pero era muy pequeño e irregular. Para esto se alquiló la maquinaria necesaria El camino que se abrió era más amplio y aunque aún no contaba con asfalto era más regular. Se dice en el pueblo que "entraron las máquinas porque los migrantes presionaron" y desde entonces "las cosas comenzaron a cambiar". Esto facilitó que se introdujera el servicio de transporte pú- 
blico, aunque sólo llegaba al poblado tres veces al día. En esta época también se incrementaron los ingresos de las familias que tenían un pariente en Estados Unidos. Todo esto tuvo un impacto significativo en la comunidad.

En 1970 se puso el asfalto al camino y aumentó la regularidad del transporte público. En el museo-casa de la cultura del pueblo hay fotografías de este evento. Isidro Cahuantzin quien fuera el agente municipal en ese tiempo disfrutó y aún disfruta del prestigio de haber sido el agente que, en opinión del pueblo, "fue quien promovió el progreso". Entre 1974 y 1975 se construyó una nueva escuela primaria. Se restauró y amplió la iglesia principal, dejando sólo a un costado la primera capilla. En esa misma década comenzó la pavimentación de la avenida y calles centrales.

En 1980 la junta del pueblo acordó que las instalaciones de la primera escuela primaria se acondicionaran para funcionar como el auditorio municipal. Se restauraron y ampliaron las instalaciones de la agencia municipal. A finales de esta década, la introducción de los servicios públicos de luz y agua potable avanzaron mucho. En el centro del pueblo se construyó una torre que desde entonces alberga el reloj. En 1990, se construyó un nuevo panteón. A lo largo de 1980 y 1990 se invirtió en pequeños negocios familiares, se abrieron más tiendas así como los molinos eléctricos de nixtamal, hoy en día esta tendencia continúa. En 2013 se pudo observar que se han abierto muchos más negocios que hace 10 años. En el año 2000 algunas familias habían comprado algunas cabezas de ganado mayor, actualmente se pudo observar que menos familias seguían criando ganado mayor. Al menos no en el área central del pueblo. Hacia 2001 se habían construido canchas de baseball y basquetbol, mismas que cuentan con vestidores y baños.

A finales de 1990 se terminó de pavimentar la avenida principal Diego Martín y de las calles paralelas Malinche y Paso de Cristo Rey. Algunas otras calles de la comunidad se pavimentaron. Actualmente casi la totalidad de las calles más céntricas están pavimentadas y cuentan con alumbrado público. Casi todas las casas cuentan con el servicio telefónico, ha aumentado la telefonía celular y servicio de internet en casa. En el año 2002 la obra más importante, desde el punto de vista de la población fue la construcción e inauguración de la nueva escuela primaria. También se han mejorado las instalaciones de la escuela primaria ya existente y de la telesecundaria. Sin duda, como ya se ha dicho, las obras más importantes de los últimos 10 años fueron dos grandes edificios perfectamente equipados en los que se inauguraron dos bachilleratos tecnológicos.

Estos cambios no habrían sido posibles sin la migración a Estados Unidos y de manera menos importante, al Distrito Federal, Puebla y Cuernavaca. A su vez esto generó una diversificación de oficios que cubren la demanda local y de otros pueblos. Ahora existe una gran oferta de servicios como albañilería, plomería, electricidad, herrería, vidriería, sastrería y más recientemente de grupos musicales. Curiosamente ante todos estos cambios intensos el gobierno del estado ha tenido una participación menor. Ciertamente durante las campañas de promoción política los candidatos de los diferentes partidos políticos han ofrecido construir obras públicas, pero según consta en las actas de la agencia municipal los trabajos se han realizado siempre con una participación de 50 ó 
$60 \%$ de las remesas y con el trabajo y mano de obra de los que permanecen en el lugar. A niveles más amplios los cambios han trascendido las fronteras. En Estados Unidos existe un organismo llamado comité-junta pro-Tlalcuapan formado, dirigido y administrado por los migrantes tlalcualpeños, el objetivo principal de este comité es satisfacer las peticiones que el pueblo les manifiesta por llamadas telefónicas, antes se les enviaba cartas, pero esto ha cambiado.

En el año 2001 en el pueblo se habían abierto tres talleres grandes donde se elaboraban cobijas las cuales se vendían en Chiautempan y dos fábricas de fibra para el lavado de trastes. En 2013 no se pudo confirmar si todavía existían estas empresas pequeñas, pero hasta 2002 ofrecían empleo a más de 100 personas, muchos de ellos niños, niñas y gente joven. Estas grandes inversiones ha sido un proceso que sin duda tomó un largo tiempo ya que los recursos provienen de empleos como cocineros (as), lavaplatos, jardineros, construcción, servicio doméstico, cajeros (as) y trabajo en el campo. Simultáneamente el fenómeno migratorio introdujo no sólo beneficios económicos sino también permitió la introducción de nuevas ideas en cuanto a la forma en que desean vivir, de sus expectativas, de las actividades que realizan y de la persona. La mejora en estos aspectos de su vida colectiva ha permitido que las familias mantengan a los niños y niñas en casa. Como se ha visto los niños siguen trabajando, ayudando pero ahora al interior de la casa a diferencia de los años de 1930 y 1960.

En el contexto de cambios profundos que ha atravesado San Pedro también existen patrones constantes, uno y el más evidente es el aprovechamiento del trabajo de los hijos en la reproducción del grupo doméstico. Si bien ahora las tareas son menos demandantes, en comparación con el pasado, aun así, los niños mientras sigan solteros deben trabajar y ayudar en beneficio de la familia. Lo que ha cambiado son las actividades que realizan. Con la introducción de las escuelas, los niños dividen ahora su rutina diaria entre las actividades escolares, el trabajo familiar que se apoya básicamente en la distribución de la ayuda de diferente índole, el trabajo asalariado y la vida comunitaria. Contrariamente a lo que se pudiera pensar, las mejoras económicas sólo han reforzado las relaciones de interdependencia entre padres e hijos.

\section{Consideraciones finales}

Como hemos podido observar en Tlalcuapan, la idea nahua local de lo que es y debe ser un niño se ha transformado en últimas 4 décadas. Gracias a la migración internacional los niños que años atrás eran dados en servidumbre fueron conservados en los hogares. Sin embargo las ideas de limpieza, orden y disciplina introducidas en el pueblo por la escuela primaria y sus programas de estudios aplicados desde el año 2000, han vuelto a poner en crisis a las familias tlalcualpeñas. Por un lado hace 4 décadas la escuela primaria contribuyó a crear en el seno de las familias la idea de que la educación escolar era una buena opción para la movilidad social. Sin embargo, en los últimos 15 años el aumento de demandas de materiales, uniformes escolares y la obligatoriedad de participar en los festivales escolares ha generado una deserción escolar importante. 
Simultáneamente, el aumento de oportunidades de trabajo asalariado para los adultos ha permitido que los hijos que no pueden asistir a la escuela los sustituyan en los trabajos domésticos y en las labores del campo. Esto nuevamente ha conllevado a la redistribución del trabajo familiar. Pese al crecimiento de una pequeña economía local el trabajo de los hijos, ahora en casa, se ha mantenido, permaneciendo una valoración positiva al hecho de que los hijos cooperen con la familia. Paralela y paulatinamente los cambios ya mencionados han introducido otra noción de infancia. Hemos encontrado que estas nociones han generado conflictos, específicamente entre la vida familiar y la vida escolar, sin embargo el mismo contexto cultural agrícola permite la negociación y re-negociación de las funciones que están permitidas para los niños, como se vio los alumnos pueden cubrir sus faltas con faenas o trabajo de mejora de las instalaciones escolares. Se pudo observar también que aunque los cambios económicos han sido importantes aún las familias con mayores ingresos ven bien que los hijos solteros, estén en México o hayan migrado continúen contribuyendo con la economía familiar. La comunidad es actualmente bombardeada por diversos medios con ideas sobre "la familia mexicana" o el modo de vivir que ven los que han migrado y comentan a sus familiares en la comunidad. Sin embargo en Tlalcuapan subsisten las prácticas locales, tales como las mayordomías, las fiestas locales. Por ejemplo, se observó que en un festival de primavera, una de las candidatas más fuertes para la corona de la Señorita Tlalcuapan, era estudiante universitaria. En favor de la candidata la gente decía que debía ganar porque era estudiante y debía ser muy inteligente. Sin embargo, en las últimas votaciones perdió porque en la opinión de la mayoría de la gente, ella era demasiado delgada y con ese cuerpo, no podría ser muy útil en el campo. Hasta el año 2013 se podía apreciar en muchos aspectos de la vida cotidiana estas contradicciones y tensiones, entre lo que la gente quiere ser y lo que puede ser. Sin embargo de forma extraordinaria siguen incluyendo lo que es útil para la comunidad y se resisten a aquello que creen que puede afectar la vida colectiva.

Se ha observado que ante ese escenario los conceptos locales y las ideas universales sobre la infancia subsisten paralelamente en dos ámbitos diferenciados: los primeros -nociones locales - se expresan en los roles sociales de la vida familiar y comunitaria. Y por otro lado las ideas universales, se viven y se expresan en la vida escolar, es decir, en el kinder, la primaria, secundaria y bachillerato tecnológico. A pesar de que subsisten actividades agrícolas y de explotación de montes, las familias campesinas, no se reconocen a sí mismas como campesinos, sino que han ido incorporando patrones de consumo más parecidos a los que se practican en las ciudades más grandes como Puebla y Tlaxcala, lo que resulta interesante de este caso es el deseo de los padres porque sus hijos tengan en sus propias palabras una mejor vida la cual, según ellos se puede alcanzar mediante la educación formal, es decir, concluir una carrera técnica, lo cierto es que estas esperanzas se desvanecen ante el costo de la educación y las demandas de materiales necesarios para mantenerse en la escuela. Considero necesario continuar con este tipo de estudios para identificar las soluciones que se generan en los hogares campesinos de Tlalcuapan para conciliar las ideas "externas" sobre 
la infancia y las ideas locales de infancia según las cuales los niños deben contribuir con el gasto familiar y la vida comunitaria la cual, para sobrevivir debe de contar con la ayuda de todos los miembros de la familia, los niños incluidos.

\section{Bibliografía}

Alzate Piedrahita, María Victoria (2002). "El descubrimiento de la infancia (I). Historia de un sentimiento". En: Revista de Ciencias Humanas. Colombia, núm. 30.

Ariés, Phillipe (1992). El niño y la vida familiar en el antiguo régimen. España: Fondo Taurus.

Baquero, Ricardo y Mariano Narodowski (1994). "Escuela y construcción de la infancia". En: Revista del Instituto de Investigaciones en Ciencias de la Educación. Año III, núm. 4, julio. México: IICE-UNAM

Bello Pérez, Isaías (1994). Tlalcuapan. Tierra donde anidan las víboras. México: SEP, SEPE.

Corona Caraveo, Yolanda (2011). "Ser niño en Tepoztlán: cuatro generaciones". Anuario de investigación. México: UAM-Xochimilco.

De Teresa Ochoa, Ana Paula. "El agro en México: un futuro incierto después de las reformas". En: La nueva relación campo- ciudad y la pobreza rural. México: INAH, UNAM Y P Y V

Durkheim, Emile (1911). "Dèbat sur l'éducation sexuelle". Colección, Clásicos de las Ciencias Sociales. Canadá: Universidad de Quebec.

Elu de Leñero, María del Carmen (1993). La luz enterrada: estudio antropológico sobre la mortalidad materna en Tlaxcala. México: Fondo de Cultura Económica.

Freud, Sigmun (1973). Tres ensayos de una teoría sexual. Madrid: Biblioteca Nueva.

Gaitán, Lourdes. (2006). Sociología de la infancia. Nuevas perspectivas. España: Síntesis.

Galeana Cisneros, Rosaura (1991). "El trabajo infantil y adolescente como instancia socializadora y formadora en, para y por la vida". Tesis. México: Departamento de Investigaciones Educativas IPN.

García Verástegui, Lía y María E. Pérez Salas (1991). Tlaxcala: Bibliografía comentada. México: Instituto Mora, Colección Fuentes.

González Jácome, Alba (2008). Humedales en el suroeste de Tlaxcala: agua y agricultura en el siglo XX. México: Universidad Iberoamericana.

Good, Catherine. (2005). "Trabajando juntos como uno: Conceptos nahuas del grupo doméstico y de la persona”. En: David Robichaux. Familia y parentesco en México y Mesoamérica: Unas miradas antropológicas. México: Universidad Iberoamericana, pp. 275-295.

Guiteras Holmes, Calixta (1972). Los Peligros del alama. México: Fondo de Cultura Económica.

Gutiérrez y Vega (1995). "Las interpretaciones, las prácticas y las reacciones sociales Del uso de solventes inhalables entre los llamados niños de la calle". En: La Niña de hoy es la mujer del mañana. Vol. 2. (1998). México: UNICEF. DIF. 
López Limón, Mercedes G. (1998). El Trabajo Infantil fruto amargo del capital. México: Edit. MGLL.

Loyo, Engracia (2006). "La educación de los indígenas. Polémica en torno de la ley de educación rudimentaria (1911- 1917)". En: Moreno, Margarita y María del Refugio González (coords.). La génesis de los derechos humanos en México. México: UNAM.

Magazine, Roger y Martha Areli Ramírez Sánchez (2007). "Continuity and change in San Pedro Tlalcuapan, Mexico. Childhood, social reproduction and transnational migration". En: Jennifer Cole y Deborah Durham G. Generations and Globalization. youth, age, and family in the new world economy. USA: Indiana University Press.

Mead, Margaret (1928). Coming age in Samoa. New York: William and Morrow Company.

Medrano Camacho, Verónica (2011). "La transformación de las perspectivas de varias generaciones de pobladores de una comunidad indígena ante el establecimiento de las instituciones". Ponencia presentada en XI Congreso Nacional de Investigación Educativa / 12. Multiculturalismo y Educación / Ponencia.

Moctezuma Pérez, Sergio (2013). "San Francisco Tepeyanco: ambiente, cultura y agricultura". Tesis de Doctorado. México: Universidad Iberoamericana.

Najunda, D. C. (2010). Contemporary studies in anthropology: A Reading. Nueva Delhi: Mittlal publication.

Nieuwenhuys, Olga (2010). “Why we need a postcolonial perspective?" En: Theorizing Childhood.

Nutini, Hugo e Isaac Barry (1974). "Los Pueblos de habla náhuatl de la región de Tlaxcala y Puebla”. México: SEP-INI, Serie Antropología Social, núm. 27.

OIT (1990). "La lucha contra el trabajo Infantil". Dirección de Assefa Bequele y Jo Boyden. Suiza: OIT.

OIT y UNICEF (1996). El Trabajo Infantil en México. México: Universidad Veracruzana.

OIT (2000). El Trabajo Infantil. México: Alfaomega.

Ortner, Sherry (1979). "¿Es la mujer con respecto al hombre lo que la naturaleza con respecto a la cultura?". En: Harris Olivia y Kate Young (comps.). Antropología y feminismo. Barcelona: Anagrama.

Piaget, Jeane (1923). The language and thought of the child. Routledge.

Rädda, Barnen (1997). Trabajo infantil en Centroamérica: El Salvador, Guatemala y Nicaragua. El Salvador: Edit. Rädda Barnen.

Ramírez Rancaño, Mario (1991). Tlaxcala una historia compartida. Siglo XX. Vol. 16. México: CONACULTA, Gobierno de Tlaxcala.

Ramírez Sánchez, Martha Areli (2007). "Helping at home: The concept of childhood and work among the Nahuas of Tlaxcala, Mexico". En: B. Hungerland, M. Liebel. Working to be someone: Child focused research and practice with working children. Londres y Filadelfia: Editores Jessica Kingsley, pp. 87-95. 
Regehr Vera, Dorothea (2005). "Estar juntos y estar aparte, en San José Aztatla. Concepciones y prácticas locales del grupo doméstico en una comunidad Mesoamericana". Tesis de Maestría. México: Universidad Iberoamericana.

Rockwell, Elsie (2007). Hacer escuela, hacer estado: la educación pos revolucionaria vista desde Tlaxcala. México: Colmex, Cinvestav.

Suárez de la Torre, Laura (1991). Tlaxcala, textos de su historia. Vol. 15. México: CONACULTA, Gobierno de Tlaxcala.

Velázquez Galindo, Yuribia (2013). “Interdependencia y Economía de dones. La 'ayuda' (Quipalehuiya) como forma económica básica entre los Nahuas, México". En: ANTIPODA, Revista de antropología y arqueología. Julio-Diciembre. Colombia: Facultad de Ciencias Sociales. Departamento de Antropología. Universidad de los Andes.

Recibido: 25/06/14

Dictaminado: 13/02/15

Corregido: 02/03/15

Aceptado: 19/03/15 